\title{
Design Scheme of Block Smart Energy Internet System
}

\author{
Li Guicun $^{1 *}$, Li Dejin ${ }^{2}$ \\ ${ }^{1}$ College of Information Engineering Baise University, Shandong Province, 533000, China \\ ${ }^{2}$ College of Information Engineering Baise University, Guigang Guangxi, 533000, China
}

\begin{abstract}
Traditional energy consumption is continuously decreasing, and new energy demand industries are continuously increasing. Full consumption of clean and renewable energy and efficient utilization of comprehensive energy will become the development trend of energy industry. The smart energy Internet based on the physical structure of the energy Internet is sweeping the world. Using smart and green to fundamentally change the existing energy structure and to adjust the structure dominated by fossil energy to a new energy structure dominated by renewable energy is of far-reaching significance to China's energy transformation and upgrading in the future. This paper aims at the design idea of smart energy Internet rack for the newly-built ChengBi campus in Baise University, combines peak-valley electricity price and photovoltaic hydrogen production technology to stabilize the fluctuation of renewable energy output, and realizes the safe and stable operation of a comprehensive energy system of distributed photovoltaic power stations. A campus smart energy Internet system is formed by "wind power + photovoltaic + energy storage + charging pile + photovoltaic hydrogen production".
\end{abstract}

\section{Introduction}

The Smart Energy Internet is a new energy utilization mode that uses the information interconnection technology to realize the deep integration of clean energy and Internet, Smart energy management is an important form of Energy Internet, block energy management and intelligent optimal scheduling system is an important and key link in the construction and operation of Smart Energy Internet[1]. The future integrated smart energy management system is a large-scale open energy management service platform supported by big data, blockchain, Internet of things, mobile Internet technology and clean energy, using block-chain technology to clarify the hierarchical and distributed structure, with the help of the cloud data Computing Center, it deals intelligently with various kinds of information such as the production, transmission and consumption of electricity, heat and cold, storage, transmission and use of various kinds of energy sources, monitor and manage the energy flow, information flow and data flow of the whole energy system, Its main characteristics include innovation of energy production mode, innovation of demand-side consumption mode, realization of energy supply-demand interaction. Smart energy management system is composed of smart energy management platform, communication system and collection terminal, As shown in figure1.

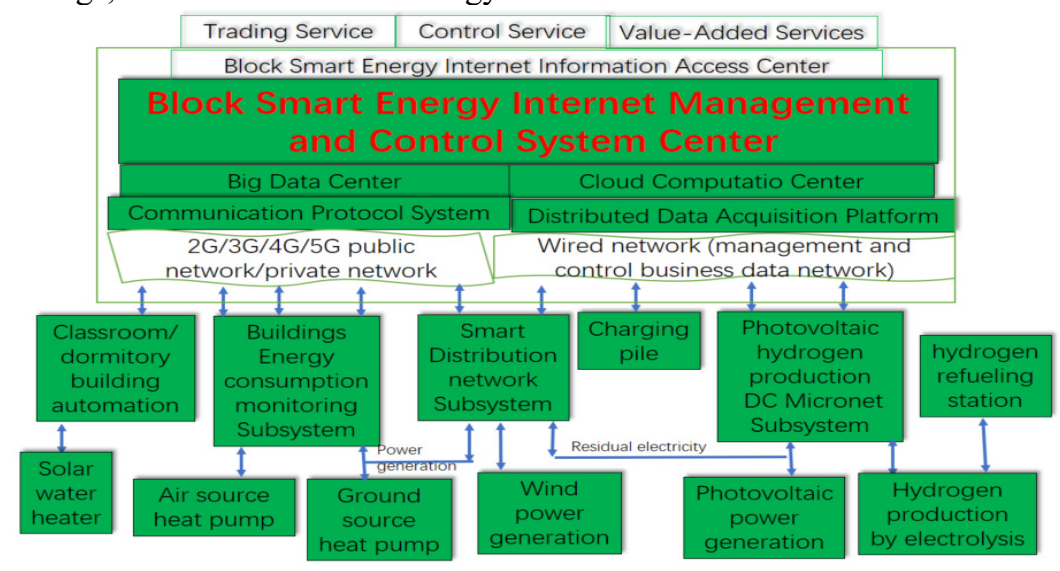

Figure 1. Schematic Diagram of Smart Energy Internet Framework

Smart Energy Internet system is a distributed interconnected system coupling energy flow, information flow and data flow. Analyzing the characteristics and processing technology of energy information is the basis

\footnotetext{
${ }^{*}$ Corresponding author's e-mail: guicunli@bsuc.cn
} 
of studying some complex problems of smart energy internet[1].Smart energy management is an important form of Energy Internet. Based on the construction of the new campus of Baise University, this paper combines the local wind resources, light resources, water resources, geothermal resources, making the ChengBi campus as a piece of the blockchain, with the ultimate goal of the Energy Internet system to reduce the cost of energy use and improve the energy structure, design and build the Smart block Energy Internet system. The initiative is in line with the new trends of the energy production, application and consumption revolution, making the use of energy in the block more low-carbon and environmentally friendly.

Given that the multi-purpose energy flow in the intelligent energy internet system, the clean energy output is difficult to predict accurately, and the operation scheduling has strong coupling and mixing characteristics, considering clean energy output volatility, the invention solves the instability of clean energy power generation and the problem of sending out, develops design idea of constructing smart DC microgrid, plans to build a new laboratory for Photovoltaic hydrogen production at Baise University.

\section{Overall design}

The overall system design is shown in figure 2, It is mainly composed of distributed wind power generation system, photovoltaic power generation system, intelligent $\mathrm{AC}$ distribution network sub-system composed of wind power and photovoltaic residual electricity, photovoltaic hydrogen generation micro-direct current network subsystem, building area energy consumption monitoring sub-system, classroom/dormitory building automation, charging pile, AC/DC load and so on. The main sections are discussed in more detail in the following sections.

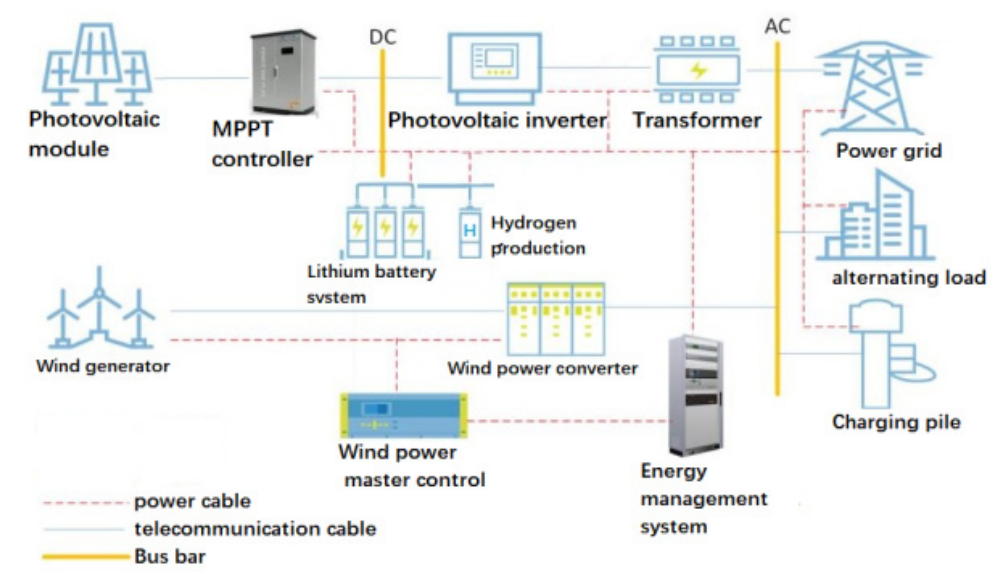

Figure 2. schematic diagram of hydrogen production, wind power and solar energy generation, Lithium battery energy storage system, transportation and charging

\section{Adoption of technical solutions}

\subsection{Distributed wind power generation}

On May 27,2017, the National Energy Administration issued the circular of the National Energy Administration on accelerating the construction of decentralized access wind power projects, no. 2017 No. 3, The document clearly states: optimizing the layout of wind power construction, accelerate the development of decentralized wind power, We will vigorously promote local use of wind power, It is an important task of wind power development during the 13th five-year plan period. The development and construction of decentralized wind power projects should be carried out in accordance with the overall principle of "overall planning, step-by-step implementation, local balance and local absorption", standardize the construction standards and clear access voltage levels, capacity and access methods and other contents [6]. And then, National and local governments have issued a series of policies and plans to promote the development of decentralized wind power. April 2018, Interim Measures for the administration of Development and construction of decentralized wind power projects issued by the State Energy Administration of China, Decentralized wind power is finally entering a new era of development. From 2018 to 2020, the government departments of Henan, Hebei and Shanxi provinces have planned more than $7 \mathrm{gw}$ of decentralized wind power construction. Societe Generale forecast, by 2020, China will have $20 \mathrm{gw}$ of distributed wind power installed, with an annual growth rate of over $100 \%$ [2]. With the development of new energy technology, the penetration rate of distributed wind power generation and distributed photovoltaic power generation (DPV) in the power grid is increasing, it also helps to adjust the voltage distribution in the distribution system. However, as the output power of clean energy power generation is affected by weather conditions, especially solar irradiance, the output power of PV may fluctuate greatly when weather conditions change, as a result, the distribution of node voltage and power flow changes, resulting in over-voltage, load shedding and other failures. Therefore, in order to solve the various problems in the utilization of new energy sources, we can consider the advantages and disadvantages of various energy sources and their complementary characteristics through the cooperative utilization of various energy sources, the Smart Energy Internet microgrid concept has 
also been proposed. Its essence is a kind of integrated intelligent energy system, which is composed of power system, electricity, heat, gas and other traditional independent energy networks, and is linked by advanced information communication technology and energy trading system [7][8]. Based on satellite images of the Baise region provided by NASA, the distribution of wind resources over the Baise region is the three large areas with good wind resources. Located on the edge of the large in the centre, the campus of Baise University, about 4.7 $\mathrm{km}$ from the ChengBi Reservoir, is an area of good wind and water resources.

Although decentralized wind power development still faces various difficulties, the maturing wind power industry is standing at a crossroads of change, the continuous innovation of Fan Technology and the rapid development of advanced technologies such as big data, blockchain, cloud computing, AI, etc [4].

On-the-spot investigation by the local meteorological department and the wind resources data provided for many years show that the wind speed is above $5 \mathrm{~m} / \mathrm{s}$ and the annual power generation hours are above 2000 hours. The installed capacity of wind farms in the area where ChengBi Campus is located can reach about $393 \mathrm{Mw}$, with an investment of 7,500 $\mathrm{RMB}(\mathrm{Kw})$, about 3 billion. The area with the best wind resources is $77 \mathrm{Mw}$, and it is estimated that $50 \mathrm{Mw}$ can be developed with an investment of 375 million RMB.

\subsection{Basic principle and characteristics of hydrogen production by solar electrolysis}

Solar electrolysis water hydrogen production is a relatively mature, the most promising way of solar hydrogen production system, through the conversion of solar energy into electrical energy, and then electrolysis of water hydrogen production. At present, photovoltaic solar power generation and water electrolysis of these two technologies are relatively mature, the formation of industry is relatively easy, especially for some regions where electricity prices are lower with abandoned photovoltaic capacity and abandoned electricity capacity.

\subsubsection{Basic principle and engineering practice of photovoltaic power generation}

A photovoltaic system consists of a solar array, a storage battery, an inverter component, a controller, and a load (see figure 2). The distributed photovoltaic system is installed on the roof of the ChengBi campus. The total area of rooftop photovoltaic generation is $32,000 \mathrm{~m}^{2}$, calculated according to $50 \%$ of the usable area (considering the factors such as maintenance space, shielding, obliquity, etc.):

320W monocrystalline silicon modules:

Length*Width(1.956m*0.996m), about2 $\mathrm{m}^{2}$,

Number of modules: $32,000 \mathrm{~m}^{2} * 0.5 / 2 \mathrm{~m}^{2}$, about 8,000. Component capacity: Take Jingke JKM355M-66H as an example, $0.355 * 80002840 \mathrm{Kw}$; module parameters consult the technical data of the manufacturer.

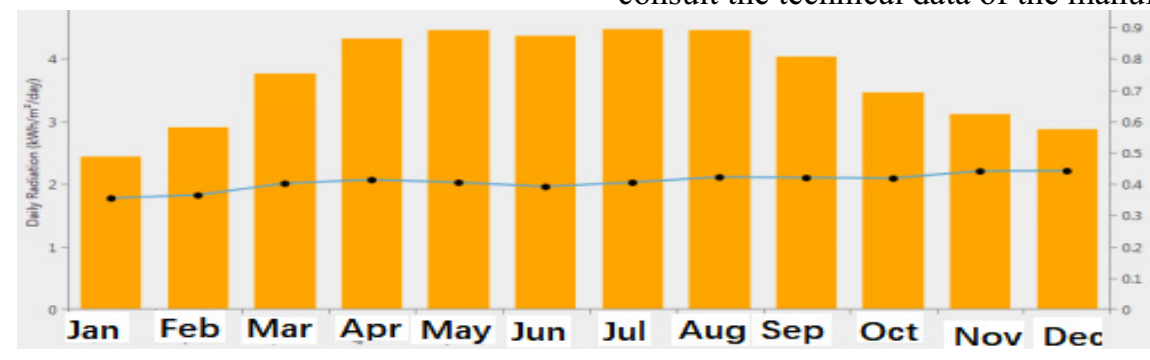

Figure 3. Schematic Diagram of solar radiation in Baise Region

As generated by HOME PRO simulation software, Baise area solar radiation resources are shown in figure 3 .

According to HOME PRO, the total number of hours of effective solar radiation in the Baise is $3.73 *$ $360=1342.8$ hours. $2840 \mathrm{Kw}$ module annual power generation: $2840 \mathrm{Kw} * 1342.8 * 85 \%=324.2$ million Kwh.

The electricity generated by solar cells is direct current, Because of the real-time change of the light intensity, the output voltage of the solar cell is also unstable. The storage battery (or the DC screen with the function of filtering and stabilizing voltage) is needed to play the role of stabilizing voltage filtering, the voltage generated by the solar cell is stabilized at the voltage required to produce hydrogen. In another sense, storage batteries can also be used to store excess power for use during peak periods. Photovoltaics, DC control and management system, and other DC load components of the intelligent DC microgrid management and control subsystem. On this basis, the new energy hydrogen production laboratory of Baise College will be established, and it is proposed to apply for the regional or national key laboratory.

\subsubsection{Basic principle of hydrogen production by electrolyzing water}

A direct current is fed into an electrolytic cell filled with water or other media (removed from the storage cell in Fig. 2 ), and the water molecules are electrochemically reacted on the electrode to decompose into hydrogen and oxygen. The chemical equation is:

$$
2 \mathrm{H}_{2} \mathrm{O}+4 \mathrm{e}=2 \mathrm{H}_{2} \uparrow+\mathrm{O}_{2} \uparrow
$$

At atmosphere and temperature, the thermodynamic voltage for electrolysis of water is usually around 3 volts. According to Faraday's first law of electrolysis, the mass of the Chemical Reaction Material occurring at the electrode interface is proportional to the current flowing through it. Faraday's second law of electrolysis states that, 
when the charge number of the elementary particle is the same, the amount of the substance that reacts on each electrode is the same, the mass of the precipitates is proportional to its molar mass. Based on the understanding of the Nernst equation, the theoretical voltage of electrolyzed water is not affected by the $\mathrm{Ph}$ of the electrolyte, the change of the ion concentration in the electrolyte and the change of the direction of the redox and the change of the electrode potential. Faraday's laws of electrolysis and Nernst equation basically laid the theoretical foundation for hydrogen production from electrolyzed water.

\subsubsection{Production Process of hydrogen production from electrolyzed water}

At present, PEM pure water electrolysis hydrogen production is one of the more commonly used water electrolysis hydrogen production technologies, Its main advantages and disadvantages are as follows;

PEM pure water electrolysis hydrogen production technology is the first U.S. nuclear submarines by the oxygen generator principle, High Purity hydrogen and oxygen can be physically separated by electrolysis of pure water in a Proton Membrane electrolyser, At present, the equipment is widely used in China, including power plant, chemical industry, metallurgy, glass, medical treatment, high-end laboratory and so on. The equipment is small, the purity of hydrogen is high, $99.9995 \%$, the whole operation is pollution-free, the operation is simple, and the remote unattended operation can be realized. The safety performance is high, the running time is long, the life of electrolytic cell is generally about 15 years. The disadvantage is that the cost of equipment is higher than that of alkaline electrolysis hydrogen production, which mainly comes from platinum catalyst. At present, various countries are studying to break through the Proton membrane platinum catalyst replacement materials, the future development can be expected. Based on the above understanding, this scheme uses PEM water electrolysis to produce hydrogen. The whole process of hydrogen production is briefly described below: High Purity hydrogen is produced by direct current electrolysis of pure water (without Alkali added). After electrification, hydrogen is produced by Cathode, oxygen is produced by anode, and hydrogen enters the hydrogen/water separator. After oxygen is collected, it can be used in physiotherapy places. A hydrogen/Water separator separates hydrogen from water. After the hydrogen enters the desiccator to dehumidify, through the pressure-stabilizing valve, the regulating valve adjusts to the rated pressure output by the outlet. The hydrogen production pressure of the cell is controlled by the sensor at a set value, and the cell power supply is cut off automatically when it reaches the set value; The power supply is restored when the pressure drops below the set value.

Compared with traditional hydrogen production methods, clean energy photovoltaic and wind power hydrogen production has several outstanding advantages. The raw materials produced are "green" and "endless". Electrolysis of water to produce hydrogen will only generate hydrogen and oxygen, which is environmentally friendly. No carbon oxygen compounds will cause pollution to the environment. Industry insiders generally believe that hydrogen as an important industrial raw material and clean fuel, in the next few decades will be a great development. Du Xiangwan, academician of The Chinese Academy of Engineering and former vice President of the Chinese Academy of Engineering, recently expressed in an exclusive interview that we want to develop green hydrogen, which can be produced from renewable energy. As a primary energy, hydrogen can not be produced without primary energy, and renewable energy is needed to develop hydrogen energy. Off the primary energy, hydrogen is water. To develop hydrogen energy, the industrial chain should focus on hydrogen production from renewable energy and identify the source [5]. The project team cooperated with well-known domestic producers of PEM pure water electrolysis to produce hydrogen, and combined with the all-day electricity price curve provided by Guangxi Electricity Market, jointly develop a control and monitoring management software based on photovoltaic generation forecasting and wind power generation forecasting to guide, monitor and control the fully automated unattended hydrogen production process to achieve real-time dynamic monitoring and control of the entire hydrogen production process, taking into account the production process of pure green hydrogen gas for photovoltaic hydrogen production and the actual situation of thermal power generation in Guangxi Autonomous Region, through overall planning for the utilization of various energy sources, taking full account of the advantages and disadvantages of various energy sources and their complementary characteristics, efforts to achieve the best cost-effective integrated energy system, intelligent energy Internet micro-network concept has been developed and improved.

\section{Conclusion}

Along with the further deepening of energy transformation and the electric power system reform, the Intelligent Energy Internet system will usher in the huge development space. The smart block energy Internet system of "wind power + photovoltaic energy + storage + charging pile + photovoltaic hydrogen production" proposed in this paper is a preliminary attempt in the new ChengBi campus of Baise University, and it is hoped that it will be continuously revised, improved, developed and promoted in the future long-term operation practice.

\section{Reference}

1. Zhang Dan, Sha Zhicheng, Zhao long, et al, Analysis and Research on Architecture of Integrated Smart Energy Management System,2017.

2. Zhou Xiaolan. Where will wind power go in the next decade?, energy, 2018, 12-05

3. Kong Dezheng. Design of inverter system in photovoltaic distributed generation. Electronic test, 
2013-07-15, 36 (8): 23-29

4. Xi Jinghua. Decentralized wind power breaks through the situation. Energy, June 5, 2018,

5. How about the domestic development of global hot "green hydrogen"? Polaris power grid 2019-06-27

6. National Energy Administration calls for accelerating the construction of decentralized wind power projects, China power enterprise management,2017-06-05

7. Liu Zhixin, Cheng Lin, Zhou Zhang, Wan Yuxiang, Dong Yuze, et al. Robust operation strategy of integrated energy system based on scene clustering analysis (), advanced technology of electrical engineering and energy, 2019-08-22 1[8] Cheng Lin, Liu Chen, Zhu ShouZhen, et al. Study of micro energy Internet based on multi energy interconnected strategy [J]. Power system technology, 2016, 40 (1): $132-138$ 